POS $\quad$ PROCEEDINGS

\title{
Search for squarks and gluinos with the ATLAS detector in final states with jets and missing transverse momentum in Run2
}

\author{
Yuta Sano* \\ Nagoya University \\ E-mail: yuta@hepl.phys.nagoya-u.ac.jp
}

Despite the absence of experimental evidence, weak scale supersymmetry remains one of the best motivated and studied Standard Model extensions. The recent increase in the center of mass energy of the proton-proton collisions at $\sqrt{s}=13 \mathrm{TeV}$ gives a unique opportunity to extend the sensitivity to production of supersymmetric particles at the Large Hadron Collider. This paper summarises the latest ATLAS result on inclusive searches for promptly decaying supersymmetric squarks and gluinos in events containing jets, missing transverse momentum and no light lepton using data of $13.3 \mathrm{fb}^{-1}$.

The 3rd International Symposium on Quest for the Origin of Particles and the Universe 5-7 January 2017

Nagoya University, Japan

* Speaker. 


\section{Introduction}

Supersymmetry (SUSY) is a generalization of space-time symmetries that predicts new bosonic partners for the fermions and new fermionic partners for the bosons of the Standard Model (SM). If the R-parity is conserved [1], squarks (including anti-squarks) and gluinos can be produced in pairs and the lightest supersymmetric particle is stable and represents a possible dark-matter candidate. Squarks and gluinos are one of the primary targets in early searches in Run2 because the large expected cross-sections are predicted for the strong production of these sparticles and the recent increase in the center of mass energy of the proton-proton collisions gives a unique opportunity to extend the sensitivity to the production at the Large Hadron Collider. The production cross section of $1.5 \mathrm{TeV}$ gluinos for $\sqrt{s}=13 \mathrm{TeV}$ is 50 times larger than that for $\sqrt{s}=8 \mathrm{TeV}$. Squarks and gluinos can decay through $\tilde{q} \rightarrow q \tilde{\chi}_{1}^{0}$ and $\tilde{g} \rightarrow q \bar{q} \tilde{\chi}_{1}^{0}$ to the lightest neutralino (Figure 1(a) and 1(b)). Additional decay modes can include the production of charginos via $\tilde{g} \rightarrow q \bar{q} \tilde{\chi}_{1}^{ \pm}$and subsequent chargino decay is $W^{ \pm} \tilde{\chi}_{1}^{0}$ (Figure 1(c)). This paper describes the result of a search for squarks and gluinos in final states containing only hadronic jets and large missing transverse momentum. The data used in this search [2] was recorded in 2015 and 2016 by the ATLAS experiment in $\sqrt{s}=13 \mathrm{TeV}$ proton-proton collisions at the LHC, corresponding to an integrated luminosity of $13.3 \mathrm{fb}^{-1}$.

(a)

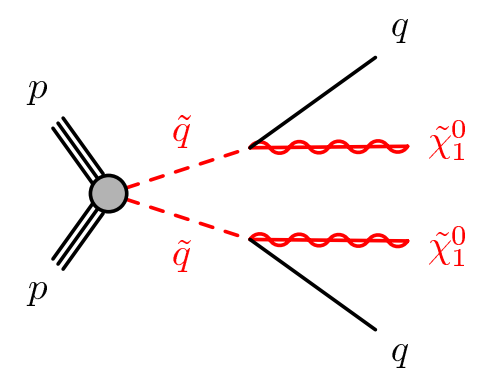

(b)

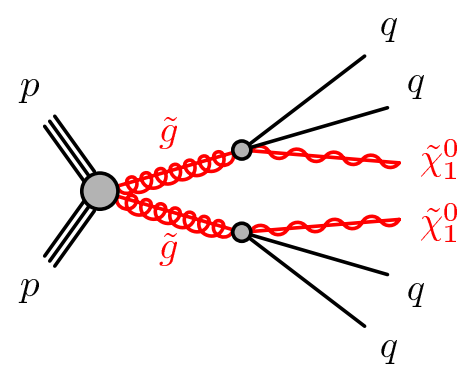

(c)

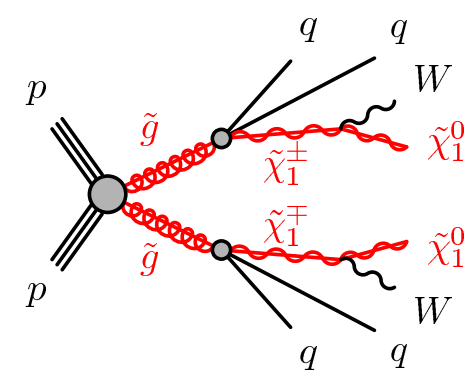

Figure 1: The decay topologies of (a) squark-pair production and (b, c) gluino-pair production, in the simplified models with (a) direct decays of squarks and (b) direct or (c) one-step decays of gluinos.

\section{Analysis strategy}

Signal Regions (SRs) to enhance each targeted signal are defined as Figure 1. The requirements on the number of jets of ' $\geq 2$ or 3 ', ' $\geq 4$ or 5 ' and ' $\geq 6$ ' target the signals in Figure 1(a), (b) and (c), respectively. The signal selection uses requirements on the $m_{\mathrm{eff}}$ (incl.) which is defined to be the scalar sum of the transverse momenta $p_{\mathrm{T}}$ of the jets with more than $50 \mathrm{GeV}$ and missing transverse energy $E_{\mathrm{T}}^{\text {miss }}$ with more than $250 \mathrm{GeV}$. The $m_{\mathrm{eff}}$ (incl.) is related to the mass of the primary SUSY particle produced.

To reduce the background from multi-jet processes, requirements on $E_{\mathrm{T}}^{\text {miss }} / m_{\mathrm{eff}}$ (or $E_{\mathrm{T}}^{\mathrm{miss}} / \sqrt{H_{\mathrm{T}}}$, where $H_{\mathrm{T}}$ is defined as the scalar sum of the transverse momenta of all jets) and $\Delta \phi$ (jet, $E_{\mathrm{T}}^{\text {miss }}$ ) which is defined to be the smallest azimuthal separation between $E_{\mathrm{T}}^{\text {miss }}$ and the momenta of any of the reconstructed jet with $p_{\mathrm{T}}>50 \mathrm{GeV}$ are designed. In the regions with at least four jets in 
the final state, additional suppression of background processes is based on the aplanarity variable which is defined as $A=3 / 2 \lambda_{3}$, where $\lambda_{3}$ is the smallest eigenvalue of the normalized momentum tensor of the jets to distinguish spherical events from planar or linear events [3].

The SM background processes contribute to the event counts in the SRs. The dominant sources in this search are $\mathrm{Z}+\mathrm{jets}, \mathrm{W}+\mathrm{jets}$, top quark pairs, single top quarks, dibosons and multi-jet production. $\mathrm{W}+$ jets, ttbar, $\mathrm{Z}+\mathrm{jets}$ and multi-jet production are fitted simultaneously to data in specific control regions defined for each of the SRs. Dibosons production is estimated entirely using MC simulation.

\begin{tabular}{|c|c|c|c|c|c|}
\hline Targeted signal & \multicolumn{5}{|c|}{$\tilde{q} \tilde{q}, \tilde{q} \rightarrow q \tilde{\chi}_{1}^{0}$} \\
\hline \multirow{2}{*}{ Requirement } & \multicolumn{5}{|c|}{ Signal Region } \\
\hline & Meff-2j-800 & Meff-2j-1200 & Meff-2j-1600 & Meff-2j-2000 & Meff-3j-1200 \\
\hline$E_{\mathrm{T}}^{\text {miss }}[\mathrm{GeV}]>$ & \multicolumn{5}{|c|}{250} \\
\hline$p_{\mathrm{T}}\left(j_{1}\right)[\mathrm{GeV}]>$ & 200 & \multicolumn{3}{|c|}{250} & 600 \\
\hline$p_{\mathrm{T}}\left(j_{2}\right)[\mathrm{GeV}]>$ & 200 & \multicolumn{3}{|c|}{250} & 50 \\
\hline$p_{\mathrm{T}}\left(j_{3}\right)[\mathrm{GeV}]>$ & \multicolumn{4}{|c|}{-} & 50 \\
\hline$\left|\eta\left(j_{1,2}\right)\right|<$ & 0.8 & \multicolumn{3}{|c|}{1.2} & - \\
\hline$\Delta \phi\left(\text { jet }_{1,2,(3)}, \boldsymbol{E}_{\mathrm{T}}^{\mathrm{miss}}\right)_{\min }>$ & \multicolumn{4}{|c|}{0.8} & 0.4 \\
\hline$\Delta \phi\left(\text { jet }_{\mathrm{i}>3}, \boldsymbol{E}_{\mathrm{T}}^{\mathrm{miss}}\right)_{\min }>$ & \multicolumn{4}{|c|}{0.4} & 0.2 \\
\hline$E_{\mathrm{T}}^{\text {miss }} / \sqrt{H_{\mathrm{T}}}\left[\mathrm{GeV}^{1 / 2}\right]>$ & 14 & 16 & 18 & 20 & 16 \\
\hline$m_{\mathrm{eff}}$ (incl.) $[\mathrm{GeV}]>$ & 800 & 1200 & 1600 & 2000 & 1200 \\
\hline
\end{tabular}

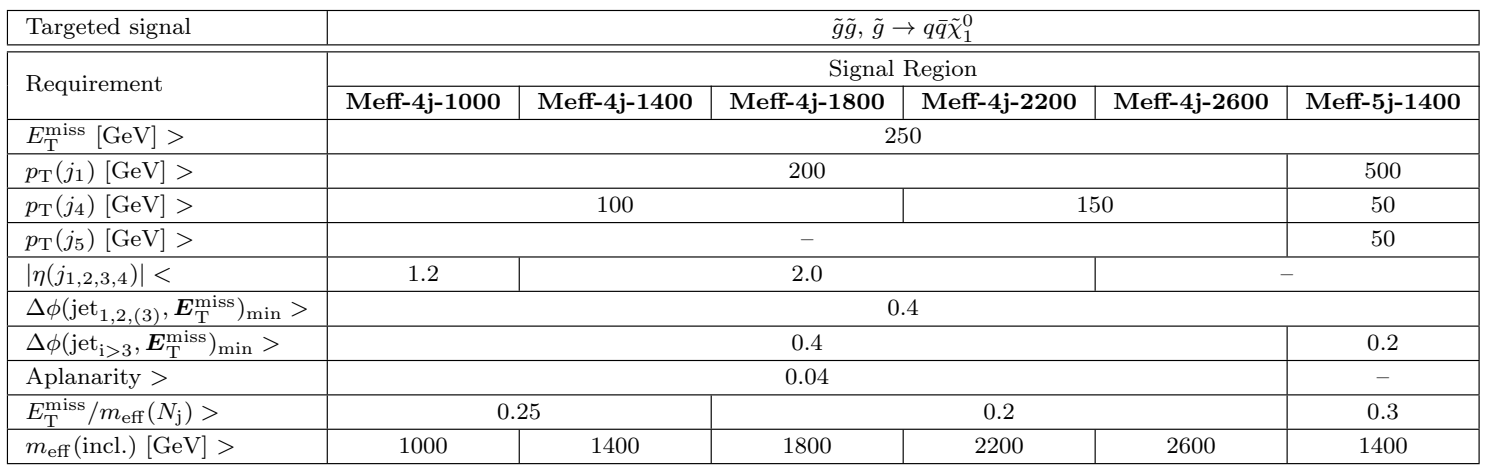

\begin{tabular}{|l|c|c|}
\hline Targeted signal & $\tilde{g} \tilde{g}, \tilde{g} \rightarrow q \bar{q} W \tilde{\chi}_{1}^{0}$ \\
\hline \hline \multirow{2}{*}{ Requirement } & \multicolumn{2}{|c|}{ Signal Region } \\
\cline { 2 - 3 } & Meff-6j-1800 & Meff-6j-2200 \\
\hline$E_{\mathrm{T}}^{\text {miss }}[\mathrm{GeV}]>$ & \multicolumn{2}{|c|}{250} \\
\hline$p_{\mathrm{T}}\left(j_{1}\right)[\mathrm{GeV}]>$ & 50 & 100 \\
\hline$p_{\mathrm{T}}\left(j_{6}\right)[\mathrm{GeV}]>$ & 2.0 & - \\
\hline$\left|\eta\left(j_{1, \ldots, 6}\right)\right|<$ & \multicolumn{2}{|c|}{0.4} \\
\hline$\Delta \phi\left(\text { jet }_{1,2,(3)}, \boldsymbol{E}_{\mathrm{T}}^{\mathrm{miss}}\right)_{\min }>$ & \multicolumn{2}{|c|}{0.2} \\
\hline$\Delta \phi\left(\text { jet }_{\mathrm{i}>3}, \boldsymbol{E}_{\mathrm{T}}^{\text {miss }}\right)_{\min }>$ & \multicolumn{2}{|c|}{0.08} \\
\hline Aplanarity $>$ & 1800 & 2200 \\
\hline$E_{\mathrm{T}}^{\text {miss }} / m_{\mathrm{eff}}\left(N_{\mathrm{j}}\right)>$ & \multicolumn{2}{|}{} \\
\hline$m_{\mathrm{eff}}($ incl. $)[\mathrm{GeV}]>$ & & \\
\hline
\end{tabular}

Table 1: Selection criteria and targeted signal model used to define signal regions. 


\section{Results}

Observed $m_{\text {eff }}$ distributions for the SR of the Meff-4j-2200 (the number of jets $\geq 4$ and $m_{\text {eff }}$ (incl.) $\geq 2200$ ) and the number of events observed in the data and the number of SM events expected to enter each of the signal regions, determined using the background-only fit are shown in Figure 2(a) and 2(b). No excess above the SM background expectation was observed in any of the SRs.

In Figure 3(a) and 3(b), limits are shown for two classes of simplified models in which only direct production of light-flavour squark and gluino pairs, corresponding to Figure 1(a) and 1(b) respectively, are considered. In Figure 3(c), limit is shown for pair-produced gluinos each decaying via an intermediate $\tilde{\chi}_{1}^{ \pm}$to two quarks, a W boson and a $\tilde{\chi}_{1}^{0}$. Squark masses below $1.35 \mathrm{TeV}$ and gluino masses below $1.86 \mathrm{TeV}$ are excluded at the $95 \%$ confidence level.

(a)

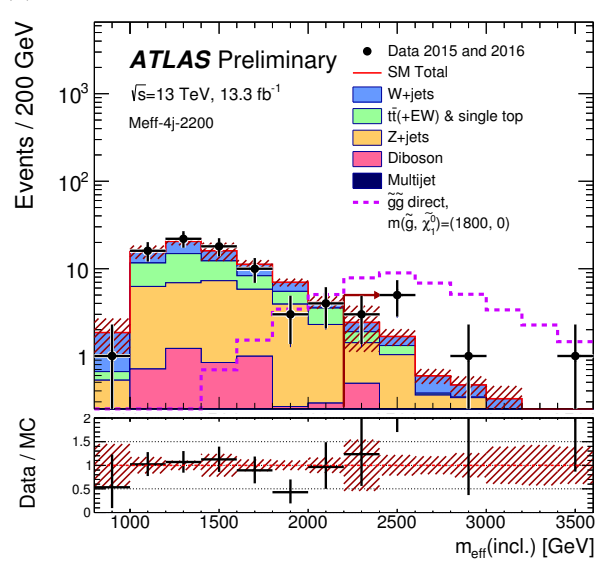

(b)

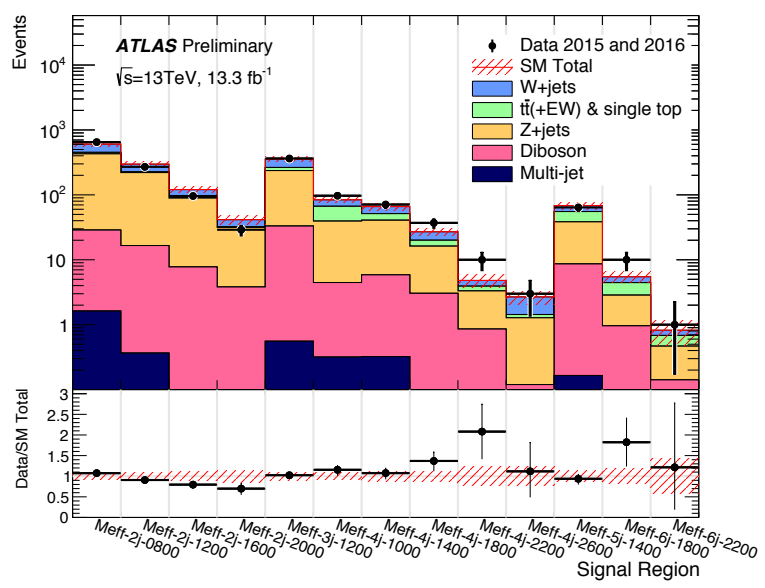

Figure 2: (a) Observed $m_{\mathrm{eff}}$ distributions for the Meff-4j-2200. (b) Comparison of the observed and expected event yields as a function of signal region.

\section{Summary}

The large expected cross-sections are predicted for the strong production of these sparticles and the recent increase in the center of mass energy of the proton-proton collisions gives a unique opportunity to extend the sensitivity to the production at the Large Hadron Collider. Squarks and gluinos in final states containing only hadronic jets and large missing transverse momentum are targeted in this analysis. SRs to enhance each targeted signal are defined. No excess above the SM background expectation was observed. Squark masses below $1.35 \mathrm{TeV}$ and gluino masses below $1.86 \mathrm{TeV}$ are excluded at the $95 \%$ confidence level. 
(a)

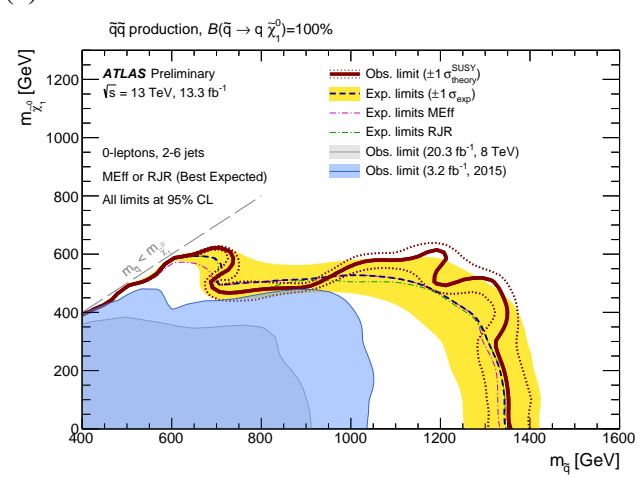

(b)

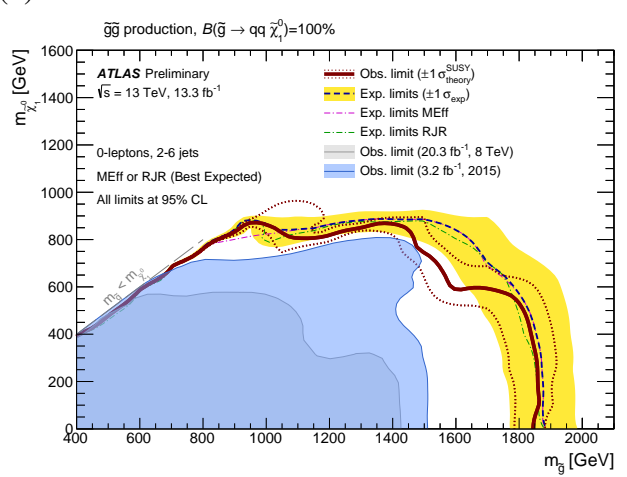

(c)

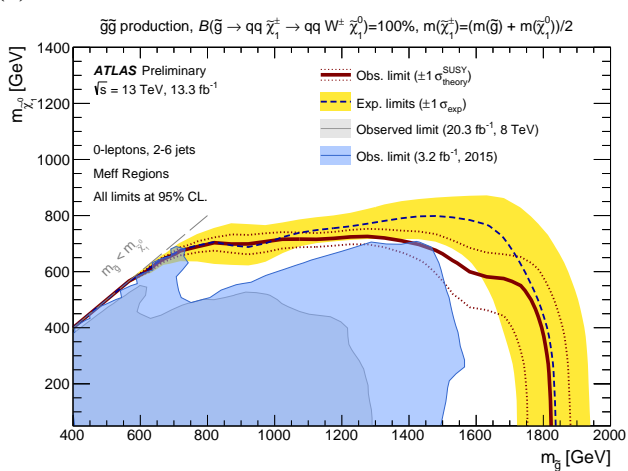

Figure 3: Exclusion limits for direct production of (a) light-flavour squark pairs with decoupled gluinos, (b) gluino pairs with decoupled squark, and (c) pair-produced gluinos each decaying via an intermediate $\tilde{\chi}_{1}^{ \pm}$ to two quarks, a W boson and a $\tilde{\chi}_{1}^{0}$ for models with a fixed $m\left(\tilde{\chi}_{1}^{ \pm}\right)=\left(m(\tilde{g})+m\left(\tilde{\chi}_{1}^{0}\right)\right) / 2$ and varying values of $m(\tilde{g})$ and $m\left(\tilde{\chi}_{1}^{0}\right)$.

\section{References}

[1] G. R. Farrar and P. Fayet, Phenomenology of the Production, Decay, and Detection of New Hadronic States Associated with Supersymmetry, Phys. Lett. B 76 (1978) 575.

[2] The ATLAS Collaboration, Further searches for squarks and gluinos in final states with jets and missing transverse momentum at $\sqrt{s}=13 \mathrm{TeV}$ with the ATLAS detector, ATLAS-CONF-2016-078

[3] C. Chen, New approach to identifying boosted hadronically decaying particles using jet substructure in its center-of-mass frame, Phys. Rev. D 85 (2012) 034007, arXiv: 1112.2567 [hep-ph]. 